\title{
Common West African HLA antigens are associated with protection from severe malaria
}

\author{
Adrian V. S. Hill, Catherine E. M. Allsopp, Dominic Kwiatkowski", \\ Nicholas M. Anstey*, Patrick Twumasi', Pamela A. Rowe ${ }^{\dagger}$, Stephen Bennett ${ }^{\dagger}$, \\ David Brewster", Andrew J. McMichael \& Brian M. Greenwood*
}

\author{
Molecular Immunology Group, Institute of Molecular Medicine, University of Oxford, John Radcliffe Hospital, Oxford OX3 9DU, UK \\ * Medical Research Council Laboratories, Fajara, The Gambia \\ † Department of Epidemiology and Population Sciences, London School of Hygiene and Tropical Medicine, London WC1E 7HT, UK \\ ¥ Royal Victoria Hospital, Banjul, The Gambia
}

A large case-control study of malaria in West African children shows that a human leucocyte class I antigen (HLA-Bw53) and an HLA class II haplotype (DRB1*1302-DQB1*0501), common in West Africans but rare in other racial groups, are independently associated with protection from severe malaria. In this population they account for as great a reduction in disease incidence as the sickle-cell haemoglobin variant. These data support the hypothesis that the extraordinary polymorphism of major histocompatibility complex genes has evolved primarily through natural selection by infectious pathogens.

THE genes of the major histocompatibility complex (MHC) which encode the human leucocyte antigens (HLA) are the most polymorphic known in man (Fig. 1) $)^{1}$. Although it was proposed that most of this variation is essentially neutral $l^{2,3}$, several observations now indicate that MHC polymorphism must be maintained by some type of selection pressure. Over the millions of years in which HLA genes have been evolving ${ }^{4,5}$, most of the current alleles should have been lost by genetic drift unless some form of balancing selection was operating ${ }^{6}$, and the unusually even distribution of HLA frequencies in human populations, without a predominance of any single allele, also signifies balancing selection pressures ${ }^{7,8}$. Furthermore, mutations which produce amino-acid substitutions cluster around the peptidebinding cleft of HLA molecules ${ }^{9-11}$ indicating that selection must have been acting on this site at some stage during evolutionary history. However, such analyses cannot show that selection is ongoing today, nor determine whether it acted through differential viability, differential fertility or some other process.

The discovery of $\mathrm{MHC}$ restriction of the immune response, whereby foreign antigens could only be recognized by $T$ lymphocytes when presented in association with class I or class II MHC molecules, led to the proposal that MHC polymorphism is maintained by different alleles providing varying degrees of protection against infectious pathogens ${ }^{12}$. An individual who is heterozygous for MHC alleles would have an increased capacity to present antigens from a range of pathogens compared with homozygotes. This theory of pathogen-driven MHC diversity requires that individuals of different $\mathrm{MHC}$ types should differ in their susceptibility to at least some major infectious pathogens. However, no convincing associations between HLA polymorphism and susceptibility to commonly fatal infectious diseases have yet been identified, in contrast to the well-known associations between HLA and many autoimmune diseases ${ }^{13}$. This has led to the proposal of many alternative theories to explain the existence of MHC polymorphism in man and other species $^{14-16}$.

We report a case-control study of the influence of HLA class I and II variation on susceptibility to Plasmodium falciparum malaria, a disease which has been a major selective force in recent human evolution ${ }^{17}$, and in which T-lymphocyte dependent mechanisms are important in providing protective immunity ${ }^{18-22}$. By detailed HLA typing of over 2,000 individuals from the West African population of The Gambia we show that an HLA class I antigen, HLA-Bw53, and an HLA class II haplotype, DRB1*1302-DQB1*0501, are associated with reduced susceptibility to severe malaria. Interestingly, both of these HLA types are much more frequent in West Africans than other racial groups, arguing that natural selection by malaria has contributed to their elevated frequencies in Africa. These data support the hypothesis that MHC polymorphism is maintained primarily by altering susceptibility to infectious pathogens.

\section{Malaria in West Africa}

$P$. falciparum malaria is a major cause of childhood mortality, currently accounting for between one half and two million deaths per year in sub-Saharan Africa alone ${ }^{23}$. In The Gambia, about $1 \%$ of children under 5 years old die each year from malaria ${ }^{24}$. It is clear that although infection by $P$. falciparum is extremely common in African children only a small proportion of these infections lead to life-threatening illness ${ }^{24}$. Many factors are likely to influence the extent of disease progression including genetically determined differences in host susceptibility. The best known host resistance gene is that encoding the $\beta$-chain of the haemoglobin $(\mathrm{Hb})$ variant, $\mathrm{HbS}$, which provides a striking illustration of natural selection by malaria ${ }^{25}$. No comparable data exist on the relevance of HLA antigen variation, although two limited studies ${ }^{26,27}$ conducted outside Africa have suggested that HLA class I alleles would be worth more detailed investigation. Piazza et al. noted some increased variation for certain
FIG. 1 Map of the location of polymorphic HLA class I and class II genes in the human major histocompatibility complex on chromosome 6 . The number of known alleles of each gene, whether identified serologically or by sequence analysis, is indicated $^{1.44}$.

Class II
Gene: $\begin{aligned} & \text { Number of } \\ & \text { alleles }\end{aligned}$

HLA genes

NATURE · VOL 352 · 15 AUGUST 1991 
HLA types between highland and lowland populations exposed in the past to different malarial endemicities in Sardinia ${ }^{28}$ but, as the authors andothers ${ }^{29}$ point out, there are several alternative explanations for these findings.

It is clear from work on the HbS polymorphism and susceptibility to malaria that the strongest protection is from severe malaria and death with less protection from mild illness and very little from parasitaemia ${ }^{25}$. To increase the probability of

TABLE 1 Gambian severe malaria study

\begin{tabular}{|c|c|c|c|}
\hline & Number & $\begin{array}{c}\text { Median } \\
\text { age }\end{array}$ & $\begin{array}{c}\mathrm{Hb} \text { AS } \\
(\%)\end{array}$ \\
\hline Severe malaria & 619 & 3.0 & 1.2 \\
\hline severe malarial anaemia & 221 & 1.8 & \\
\hline cerebral malaria & 429 & 3.6 & \\
\hline Mild controls & 510 & 1.9 & 12.9 \\
\hline Mild malaria & 354 & 3.0 & 2.8 \\
\hline Severe controls & 332 & 2.2 & 10.9 \\
\hline Healthy adults & 220 & 28 & 16.8 \\
\hline
\end{tabular}

Number of individuals recruited in each clinical category, median age and $\mathrm{HbS}$ carrier (=Hb AS genotype) rate. The HbAS percentages are based on results from 1,802 of the 2.035 individuals (S. Abdalla et al., manuscript in preparation). The carrier rate in the children with severe malaria $(1.2 \%)$ was significantly lower $\left(\chi_{1}^{2}=52.5, P<10^{-11}\right)$ than in the mild controls $(12.9 \%)$. The adults had a higher HbAS frequency than both the mild $\left(\chi_{1}^{2}=1.1\right)$ and severe $\left(\chi_{1}^{2}=3.4, P=0.07\right)$ control children, which is only in part explained by the small expected increase in carrier rate with age as a result of differential mortality from malaria ${ }^{25}$. Design: Children up to the age of 10 years were recruited at the Royal Victoria Hospital, Banjul, and the Medical Research Council Hospital, Fajara, 8 miles away, during the months of maximum malaria transmission ${ }^{30}$ from August to November in 1988 and 1989, and aiso during August and September of 1990. Approval for the study was obtained from the Gambian government/MRC joint ethical committee and permission for venesection from a parent or guardian. A diagnosis of malaria was made if a child with an appropriate clinical picture had a parasitaemia $>2,500 \mu \mathrm{l}^{-1}$, a level likely to be associated with illness in this setting, and relevant clinical and laboratory investigations excluded other diagnoses. Malaria cases were assigned to the severe group ${ }^{31,68,69}$ if any of the following features were present: (1) cerebral malaria, unrousable coma of score $<3$ (by standard criteria ${ }^{69}$ ) or repeated prolonged ( $>30 \mathrm{~min}$ ) seizures; (2) severe malarial anaemia, haemoglobin level $<5 \mathrm{~g} \mathrm{dl}^{-1}$ on admission; (3) hypoglycaemia, blood glucose level $<2.2 \mathrm{mmol}$. Fifty-six children had both cerebral malaria and severe malarial anaemia and 25 had hypoglycaemia alone. Of the 88 children who died, 72 had cerebral malaria, four had severe malarial anaemia and 12 had both: a further 33 were discharged with residual neurological deficits. All children fulfilling the operational definition of severe malarial anaemia had malaria, and several factors indicate that this was the major cause of their anaemia despite the low background haemoglobin levels of normal children in The Gambia ${ }^{68}$. These are their high spleen and gametocyte rates, normochromic normocytic blood films, their very low HoS carrier rate (sickle cell homozygotes were excluded), and the striking concordance of the seasonality of this condition with that of cerebral malaria in The Gambia ${ }^{30}$. Hence, although occasional cases may have had additional factors contributing to the anaemia, malaria was the major cause of the life-threatening state. As expected ${ }^{69,70}$, parasite densities (geometric mean with $95 \% \mathrm{ci} \times 10^{3} \mu \mathrm{l}^{-1}$ ) correlated weakly with severity: cerebral malaria $(84.9,69.5-103.7)$. mild malaria $(70.5$, $60.8-81.8)$, and in the more chronically infected severe malarial anaemia group (61.5 39.6-95.4). Although parasite densities were higher among those who died (122.4, $85.2-175.8)$ than survivors $(70.7,62.7-79.6)$, the fairly weak correlation between parasite density and clinical severity probably relates to numerous factors including the higher rate of prior antimalarial administration among the severe $\operatorname{cases}^{70}$, the greater importance of sequestered than peripheral blood parasites ${ }^{31}$ and the complex dynamics of $P$. falciparum parasitaemias ${ }^{11}$. The cases of severe malaria were matched, as a group, to the other three groups of children for two variables: age and area of residence around Banjul. The 'mild controls' (see text) were recruited at both hospitals and at health centres in the study area. 'Severe controls' were inpatients at the two hospitals with a large range of other acute, mainly infectious, illnesses, but without evidence of current or recent malarial infection. These controls were considered separately from the mild (outpatient) controls because (ref. 72 and above data) they may be more susceptible to malaria than the general population, as found elsewhere in Africa ${ }^{72}$ : this effect may arise from an increased likelihood of children who are susceptible to frequent malarial episodes becoming severely ill during other infections, for example because of malaria-induced immunosuppression. Children with unexplained anaemia and known $\mathrm{HbS}$ homozygotes were excluded as controls. Two HbSS individuals were found among 603 severe malaria cases and 6 of 254 mild controls analysed were found to be previously unsuspected HbSS homozygotes. The ethnic composition of the population in this area is mixed: Mandinka (42\%), Jola (14\%), Wolof (14\%), Fula $(12 \%)$ and several less common ethnic groups. The children were not matched for ethnic group: instead analyses stratified for this variable were done. HLA allele frequency differences between the ethnic groups were small and the proportions of each ethnic group among the clinical categories were found to be very similar. The healthy adults were male blood donors matched retrospectively to the children for ethnic group. detecting a significant effect of HLA variation we studied children with very severe illness, the majority of whom would have died without treatment. Most malarial deaths in African children are attributable to severe malarial anaemia or cerebral malaria ${ }^{30,31}$. Although the precise pathophysiological mechanisms of these two major complications are unknown, it appears that severe malarial anaemia is often associated with failure to clear parasites from the circulation so that continuing red cell destruction leads to severe anaemia, whereas cerebral malaria is associated with blockage of cerebral blood vessels by parasitized erythrocytes ${ }^{32}$. We therefore consider each of these conditions separately, particularly for the analysis of HLA class II antigens which, through their influence on antibody formation and hence parasite clearance ${ }^{33}$, might be expected to have a larger effect on severe malarial anaemia.

From 1988-90 we studied more than 600 cases of severe malaria (Table 1), severity being defined by standard clinical criteria which are powerfully predictive of outcome, at least for cerebral malaria. Children with severe malarial anaemia had a malarial illness with an extremely low and life-threatening haemoglobin level $\left(<5 \mathrm{~g} \mathrm{dl}^{-1}\right)$. Hence most of these children, like those with cerebral malaria, were likely to have died without treatment even though, because of the effectiveness of transfusion therapy, few of the 88 hospital deaths were among this group. The severe malaria cases were compared with a large group of control children, frequency matched to the cases for age and area of residence. These 'mild controls' were children with mild, mostly infectious, illnesses who did not require hospital admission and did not have any malarial parasites in their blood on microscopy. Samples were also collected from three further groups to assist in the interpretation of any association seen in the primary comparison and to address particular issues. These were children with 'mild malaria', healthy adult blood donors and children hospitalized with illnesses other than malaria, 'severe controls', as defined in Table 1.

Determination of the carrier rates for $\mathrm{HbS}$ was performed to provide a measure against which any protective effect of HLA types could be assessed. The expected low frequency of carriers of $\mathrm{HbS}$ was found in the children with severe malaria $(1.2 \%)$ compared with the mild controls $\left(12.9 \%, \chi_{1}^{2}=52.5, P<1 \times\right.$ $\left.10^{-11}\right)$, the relative risk $(R R)$ of $0.08(95 \%$ confidence intervals (ci) $0.04-0.16$ ) indicating that children who are carriers of $\mathrm{HbS}$ have more than $90 \%$ protection from severe malaria (Table 1 ).

\section{HLA class I antigens}

HLA class I antigens present peptides to cytotoxic T cells which, in certain mouse models of malaria, are induced by sporozoite infection and provide effective immunity by acting against the liver stage of the malaria parasite ${ }^{19-22}$. To look for an association between HLA class I antigens and susceptibility to malaria in man we adopted a two-stage strategy. Initially we determined the HLA class I types of roughly half the cases of severe malaria and controls serologically (Table 2). Although both HLA-A24 and HLA-B14 were more common among the cases of severe malaria, their frequencies were low and the differences were of marginal statistical significance. The frequency of HLA-Bw53, a common antigen in this population, was significantly decreased $(15.7 \%)$ among the cases of severe malaria compared with either the mild controls $\left(24.3 \%, \chi_{1}^{2}=4.3, P=0.04\right)$, all the control children $\left(22.9 \%, \chi_{1}^{2}=4.7, P=0.03\right)$, or the healthy adults $(25.0 \%$, $\left.\chi_{1}^{2}=4.2, P=0.04\right)$. The decrease in frequency was apparent both for cases of severe malarial anaemia $(14.7 \%)$ and of cerebral malaria $(16.1 \%)$

However, because in this serological study we have tested for an association between susceptibility to malaria and 45 different HLA class I antigens, a single HLA association may have turned up simply by chance as a result of making numerous comparisons. So, as stage two, we retested the association on the remaining unanalysed samples asking a single question: is the frequency of HLA-Bw53 lower among the cases of severe 
TABLE 2 HLA class I antigen frequencies

\begin{tabular}{|c|c|c|c|c|c|c|c|c|}
\hline & \multicolumn{2}{|c|}{$\begin{array}{l}\text { Severe } \\
\text { malaria } \\
n=306\end{array}$} & \multicolumn{2}{|c|}{$\begin{array}{c}\text { Mild } \\
\text { controls } \\
n=144\end{array}$} & \multicolumn{2}{|c|}{$\begin{array}{l}\text { Severe } \\
\text { controls } \\
n=171\end{array}$} & \multicolumn{2}{|c|}{$\begin{array}{c}\text { Healthy } \\
\text { adults } \\
n=112\end{array}$} \\
\hline & Number & $\%$ & Number & $\%$ & Number & $\%$ & Number & $\%$ \\
\hline A1 & 41 & 13.7 & 13 & 9.0 & 22 & 12.9 & 17 & 15.2 \\
\hline A2 & 74 & 24.2 & 41 & 28.5 & 46 & 26.9 & 32 & 28.6 \\
\hline A3 & 27 & 8.8 & 8 & 5.6 & 21 & 12.3 & 7 & 6.3 \\
\hline A23 & 85 & 27.8 & 32 & 22.2 & 43 & 25.1 & 26 & 23.1 \\
\hline A24 & 6 & 2.0 & 0 & 0 & 0 & 0 & 0 & 0 \\
\hline A26 & 44 & 14.4 & 25 & 17.4 & 26 & 15.2 & 13 & 11.6 \\
\hline A28 & 53 & 17.3 & 35 & 24.3 & 34 & 19.9 & 16 & 14.3 \\
\hline A29 & 12 & 3.9 & 7 & 4.9 & 5 & 2.9 & 3 & 2.7 \\
\hline $\mathrm{A} 30$ & 73 & 23.9 & 29 & 20.1 & 34 & 19.9 & 31 & 27.7 \\
\hline A31 & 1 & 0.3 & 2 & 1.4 & 1 & 0.6 & 1 & 0.9 \\
\hline A32 & 24 & 7.8 & 8 & 5.6 & 8 & 4.7 & 10 & 8.9 \\
\hline Aw33 & 67 & 21.9 & 33 & 22.9 & 39 & 22.8 & 29 & 25.9 \\
\hline Aw34 & 11 & 3.6 & 6 & 4.2 & 5 & 2.9 & 3 & 2.7 \\
\hline Aw36 & 1 & 0.3 & 0 & 0 & 0 & 0 & 1 & 0.9 \\
\hline B7 & 41 & 13.4 & 17 & 11.8 & 20 & 11.7 & 17 & 15.2 \\
\hline B8 & 67 & 21.9 & 31 & 21.5 & 26 & 15.2 & 18 & 16.1 \\
\hline B13 & 3 & 1.0 & 2 & 1.4 & 1 & 0.6 & 0 & 0 \\
\hline B14 & 30 & 9.8 & 5 & 3.5 & 14 & 8.2 & 4 & 3.6 \\
\hline B15 & 6 & 2.0 & 7 & 4.5 & 4 & 2.3 & 0 & 0 \\
\hline B17 & 68 & 22.2 & 27 & 18.8 & 39 & 22.8 & 24 & 21.4 \\
\hline B418 & 20 & 6.5 & 7 & 4.9 & 6 & 3.5 & 6 & 5.4 \\
\hline B22 & 9 & 2.9 & 2 & 1.4 & 8 & 4.7 & 2 & 1.8 \\
\hline B27 & 13 & 4.2 & 5 & 3.5 & 2 & 1.2 & 3 & 2.7 \\
\hline B35 & 86 & 28.1 & 48 & 33.3 & 53 & 31.0 & 32 & 28.6 \\
\hline B37 & 2 & 0.7 & 1 & 0.7 & 0 & 0 & 3 & 2.7 \\
\hline B39 & 6 & 2.0 & 4 & 2.8 & 6 & 3.5 & 2 & 1.8 \\
\hline B40 & 5 & 1.6 & 1 & 0.7 & 1 & 0.6 & 2 & 1.8 \\
\hline Bw41 & 9 & 2.7 & 4 & 2.8 & 6 & 3.5 & 2 & 1.8 \\
\hline Bw42 & 16 & 5.2 & 5 & 3.5 & 7 & 4.1 & 7 & 6.3 \\
\hline B44 & 12 & 3.9 & 1 & 0.7 & 8 & 4.7 & 4 & 3.6 \\
\hline B45 & 13 & 4.2 & 5 & 3.5 & 3 & 1.8 & 3 & 2.7 \\
\hline B49 & 25 & 8.2 & 13 & 9.0 & 14 & 8.2 & 11 & 9.8 \\
\hline Bw50 & 14 & 4.6 & 4 & 2.8 & 10 & 5.8 & 5 & 4.5 \\
\hline B51 & 17 & 5.6 & 7 & 4.9 & 7 & 4.1 & 4 & 3.6 \\
\hline Bw52 & 2 & 0.7 & 1 & 0.7 & 1 & 0.6 & 0 & 0 \\
\hline Bw53 & 48 & 15.7 & 35 & 24.3 & 37 & 21.6 & 28 & 25.0 \\
\hline Bw70 & 46 & 15.0 & 22 & 15.2 & 22 & 12.9 & 19 & 17.0 \\
\hline Cw1 & 15 & 4.9 & 8 & 5.6 & 9 & 5.3 & 6 & 5.4 \\
\hline Cw2 & 50 & 16.4 & 17 & 11.8 & 21 & 12.3 & 24 & 21.4 \\
\hline Cw3 & 91 & 29.8 & 52 & 36.1 & 54 & 31.6 & 33 & 29.5 \\
\hline Cw4 & 80 & 26.2 & 40 & 27.8 & 52 & 30.4 & 33 & 29.5 \\
\hline Cw5 & 20 & 6.6 & 7 & 4.9 & 6 & 3.5 & 6 & 5.4 \\
\hline Cw6 & 43 & 14.1 & 23 & 16.0 & 23 & 13.5 & 16 & 14.3 \\
\hline Cw7 & 55 & 18.0 & 17 & 11.8 & 25 & 14.6 & 19 & 17.0 \\
\hline Cw8 & 26 & 8.5 & 5 & 3.5 & 11 & 6.5 & 4 & 3.6 \\
\hline
\end{tabular}

Number of individuals with each HLA class I antigen and the percentage antigen frequencies in the groups defined in Table 1 . Significant frequency differences for individual antigens are described in the text. Subdivision of individuals with the HLA-Bw53 antigen into heterozygotes and apparent homozygotes showed the following numbers of the latter in each category: severe malaria, 11; mild controls, 6; severe controls, 5 ; adults, 4 . Thus, there is no evidence of a significant difference in the relative risks of severe malaria between heterozygotes and apparent homozygotes for HLABw53. The frequencies of individuals apparently homozygous for HLA-A, $-B$ and $-C$ alleles were also compared in these groups. A significantly lower frequency of HLA-B homozygotes was found in the severe malaria group, $17.3 \%$, compared with the pooled control children, $25.5 \%\left(\chi_{1}^{2}=5.7, P=0.017: \mathrm{M}-\mathrm{H}\right.$ for ethnic group $\chi^{2}=5.9, P=0.015$. $R R=0.60(0.40-0.91))$. No significant difference was found in HLA-A or -C homozygosity rates although the latter are particularly affected by a high frequency of serologically undetected alleles. Statistical analysis: In all tables this was done using the $\chi^{2}$ test with continuity corrections. Allowance for confounding factors was made by the Mantel-Haenszel test and by logistic regression ${ }^{56}$. Since severe malaria is comparatively rare, the relative risk $(R R)$ may be estimated by the odds ratio; this is given with an associated $95 \%$ confidence interval. The problem of multiple comparisons was dealt with for HLA class I antigens by a two-stage strategy, and for class II haplotypes by multiplying each $P$-value by the number of concurrent tests performed. Serological types were determined by the standard microlymphocytotoxicity assay using 180 well-characterized antisera on fresh or cryopreserved cells. From the total number of children entered into the study those for serological typing were chosen randomly, limited only by the availability of sufficient viable lymphocytes. More severe controls were included than mild controls because only tiny volumes of blood were available from most of the latter. The following specificities were tested for but not found: HLA-A11, -A25, -Aw43, -B38, -Bw47, -Bw48 and -Bw73. malaria than in the mild controls? To do this, we cloned and sequenced the HLA-Bw53 allele ${ }^{34,35}$ and devised a method of typing for this sequence using allele-group-specific amplification by the polymerase chain reaction (PCR) and oligonucleotide hybridization. Using the PCR method to test the remaining samples (Table 3), the frequency of HLA-Bw53 was $16.9 \%$ among the cases of severe malaria and $25.4 \%$ in the mild controls $\left(\chi_{1}^{2}=6.4, P=0.008\right.$; Mantel-Haenszel test $(\mathrm{M}-\mathrm{H})$ for ethnic group $\chi_{1}^{2}=6.4, P=0.01, R R=0.61$ ), confirming the association suggested by the serological results. Combining the serological and PCR data the relative risk of severe malaria among individuals with HLA-Bw53 compared with those without this allele is $0.59(0.43-0.81)$. We also used the PCR method to type the remaining adult controls who again showed a frequency of about $25 \%$, and the cases of mild malaria who had a lower, but not significantly different, frequency of $22.6 \%$ (Table 3 ).

The HLA-Bw53 antigen is present at highest frequency in sub-Saharan Africa: in $40 \%$ of Nigerians ${ }^{36}, 25 \%$ of Gambians, $21 \%$ of Zambians ${ }^{37}$ and $16 \%$ of Zimbabweans ${ }^{38}$, but is much less frequent in black South Africans $(2 \%)^{39}$. It is rare in caucasian and oriental populations $(0-1 \%)^{40,41}$ and apparently absent in Pacific islanders ${ }^{42}$ and Amerindians ${ }^{41}$. Hence, this HLA class I allele, which is associated with significant protection from severe malaria, has many similarities in its global distribution to the classical protective haemoglobin variant, $\mathrm{HbS}$ (ref. 25).

\section{HLA class II haplotypes}

The importance of antibodies in defence against the blood stage of malaria infection has been demonstrated in several ways $^{18,33,43}$. So, to look for an HLA class II association with

TABLE $3 \quad$ HLA-Bw53 association with severe malaria

\begin{tabular}{|c|c|c|c|c|}
\hline & \multicolumn{2}{|c|}{ Serology } & \multicolumn{2}{|r|}{ PCR } \\
\hline & No. & HLA-Bw53 (\%) & No. & HLA-Bw53 (\%) \\
\hline Severe malaria & 306 & 15.7 & 307 & 16.9 \\
\hline Mild controls & 144 & 24.3 & 364 & 25.4 \\
\hline Mild malaria & - & - & 353 & 22.6 \\
\hline Healthy adults & 112 & 25.0 & 106 & 26.4 \\
\hline
\end{tabular}

HLA-Bw53 antigen frequencies determined by serology (Table 2 ) and by PCR. There is no overlap between the samples analysed by the two methods. Analysis of interactions: Of the seven individuals heterozygous for $\mathrm{HbS}$ among the severe malaria cases, $1(14.3 \%)$ had the HLA-Bw53 antigen: $97(16.6 \%)$ of 586 cases of severe malaria with the $\mathrm{Hb}$ AA genotype had this antigen. Analysis of a possible interaction between HLA-Bw53 and the HLA class II haplotype, DRw13.02-DQw1, associated with protection from severe malarial anaemia (Table 4), showed that, of the 34 individuals with DRw13.02 in the severe malarial anaemia group (out of 216 typed for both antigens), $4(11.8 \%)$ had HLA-Bw53. Of the 138 mild controls with DRw13.02 (out of 477 typed for both antigens), $39(28.3 \%$ ) had HLA-Bw53: RR of severe malarial anaemia in individuals with both antigens compared to individuals with neither $=0.21(0.05-0.60)$, which is similar to the product of the individual antigen relative risks. Similar analysis of the HLA-Bw53/DRw13.02 interaction among all severe malaria cases also showed no significant interaction. Hence, although the numbers with both antigens are relatively small, there does not appear to be any strong epistatic interaction between these alleles. Analysis of parasite densities: Because of numerous confounding factors, such as the high rate of prior treatment particularly among severe cases ${ }^{70}$, this study could only have detected very large effects of genetic factors on peripheral blood parasite density: to avoid increasing the number of comparisons assessed, and thereby reducing the power of the study, this supplementary analysis was not part of the study design. Although the parasite density (geometric mean with $95 \% \mathrm{ci} \times 10^{3} \mu \mathrm{I}^{-1}$ ) among malaria cases (studied within $2 \mathrm{~h}$ of arrival at hospital, $n=523$ ) with HL.A-Bw53 (72.6, 55.9-94.3) was lower than in those without this antigen $(77.1,67.7-87.8)$ neither this difference (Wilcoxon test: $P=0.76$ ) nor the larger reduction in mean parasite density among those with HLA-DRw13.02 (68.7.54.2-87.1) compared with those without (81.1. 71.0-92.6; $P=0.23$ ) was statistically significant. PCR typing was done for HLA-Bw53 as described elsewhere ${ }^{73}$. Briefly, the primers 5'-CCGGAACACACAGATCTT-3' and 5'GTCGTAGGCGGACTGGTC-3' were used to specifically amplify only HLA-Bw53 and HLAB35 in a PCR buffer containing $10 \%$ DMSO and $1 \mathrm{mM} \mathrm{MgCl}$, with 35 cycles of denaturation at $94^{\circ} \mathrm{C}(90 \mathrm{~s})$, annealing at $58{ }^{\circ} \mathrm{C}(120 \mathrm{~s})$ and extension at $72{ }^{\circ} \mathrm{C}(120 \mathrm{~s})$. HLA-Bw53 alleles were distinguished from HLA-B35 by hybridization with an oligonucleotide probe $5^{\prime}$-CGGATCGCGCTCCGCTAC- $3^{\prime}$ to the $3^{\prime}$ end of exon 2, with washing in $6 \times \operatorname{SSC}$ at $59{ }^{\circ} \mathrm{C}$, after immobilization of the amplification product on nitrocellulose filters. 
susceptibility to the two forms of severe malaria, severe malarial anaemia and cerebral malaria, we determined HLA class II haplotypes defined by restriction fragment length polymorphisms (RFLP) of the DRB and DQB genes, a molecular method of HLA typing that has some advantages over alternative techniques. Analysis (Table 4) showed a highly significant difference in frequency for a single haplotype, with the X-I RFLP pattern, between the children with severe malarial anaemia and the mild controls. This haplotype pattern is invariably associated with the DRw13 and DQw1 serological specificities and subtyping by DNA sequencing and T cell assays has shown the DRw13 allele to be of the DRB1*1302 subtype ${ }^{44-48}$. This was confirmed for this Gambian population by sequencing of the DRB1 gene and typing of many further samples by allele-group-specific PCR and oligonucleotide hybridization ${ }^{49}$ (Table 4). Like HLA$\mathrm{Bw} 53$, this haplotype is less frequent among the cases of severe malarial anaemia ( $8.7 \%$ gametic frequency) than in the mild controls $\left(16.4 \%, \quad \chi_{1}^{2}=14.0, \quad P=0.00018, \quad P_{\text {corrected }}=0.0023\right)$, indicating that it is a protective rather than a susceptibility haplotype. Statistically significant protection was seen only from severe malarial anaemia, even though the frequency of this haplotype was also decreased in the children with cerebral and mild malaria compared with the mild controls. Analysis of genotype frequencies showed that, for each form of severe malaria, homozygotes for this haplotype had lower relative risks than heterozygotes (Table 4).

In almost all cases reported previously, the DRB1* 1302 allele has been linked to the DRB3 ${ }^{*} 0301$ allele ${ }^{50}$ and to DQ genes specifying the DQw6 (rather than the DQw5) subtype of DQw1 (ref. 51). However, sequencing of the DQA1 and DQB1 genes from a Gambian with a DRB1*1302 haplotype showed a novel arrangement of allelic sequences (Fig. 2): a DQB ${ }^{*} 0501$ rather than the expected DQB1*0604 sequence was linked to the usual DQA1 sequence ${ }^{52}$. Furthermore, this DRw13-DQw5 haplotype is more common in The Gambia than the caucasian DRw13DQw6 haplotype (Fig. 2). Comparison of the protective efficacies of the two haplotypes against severe malarial anaemia showed that the associated relative risks were similar, suggesting that both are protective and that the DRB1*1302 and DRB3*0301 alleles that they have in common may be more important than the DQB gene for protection from severe malarial anaemia. However, although the more common DQw5 haplotype is clearly associated with protection, the less frequent DQw6 haplotype is difficult to assess with certainty, precluding a definite assignment of the association to particular DR or DQ genes. The protective DQw5 haplotype is probably the same as the DRw13-DQw5 haplotype found in American blacks ${ }^{45,48}$ and an Israeli cell line ${ }^{53}$, but this haplotype is clearly very rare in northern Europeans ${ }^{54}$ and north American caucasians ${ }^{55}$. There was no significant ${ }^{56}$ linkage disequilibrium between HLA-Bw53 and $-\mathrm{DRB} 1 * 1302$ in the population as a whole $(\Delta=0.001, P>$ 0.7 ), nor within clinical groups, indicating independent associations. Although this study was not designed to measure the influence of HLA antigens on peripheral blood parasite densities, retrospective examination of the available data showed no large differences (see Table 3). Analysis of the relative risks of severe malaria among individuals with more than one of the protective alleles, HbS, HLA-Bw53 and DRB1*1302 (see legends to Tables 2-4), showed no clear evidence of epistatic interactions, but only substantial effects would have been detectable.

\section{HLA and protective immunity}

The simplest explanation for these associations, amongst several possibilities, is that they relate to the antigen-presenting ability of HLA molecules. Thus, the association between HLA-Bw53 and protection from severe disease would suggest that, as in animal models ${ }^{19-22}$, class I-restricted T lymphocytes, presumably cytotoxic T cells (CTL), play an important part in providing protective immunity to malaria in man. Because HLA class I molecules are absent on human red blood cells, these CTL would probably act against the liver-stage malaria parasite. Similarly, a plausible explanation for the HLA class II association is that only individuals with this haplotype are able to present a particular conserved epitope on a blood-stage parasite antigen to helper $\mathrm{T}$ cells, leading to more rapid parasite clearance. It may be surprising that an infectious organism as large as a malaria parasite should give rise to a disease that is HLAassociated: $P$. falciparum must contain thousands of potential T-cell epitopes which will have varying HLA restriction patterns, and immune responses against many $P$. falciparum epitopes are indeed evoked ${ }^{57}$. The existence of an HLA association implies that most such responses must be of limited protective value, and that the number of epitopes to which a strongly protective
FIG. 2 Top, Gene maps of the two DRB1*1302 haplotypes found in The Gambia. Bottom, Percentage frequencies of the two DRB1*1302 haplotypes in the various groups. The frequencies of these two haplotypes sum to the frequency of the DRw13.02-DQw1 haplotype given in Table 4. The DQw5 haplotype was significantly less frequent among the cases of severe malarial anaemia compared with the mild controls $\left(\chi_{1}^{2}=7.9\right.$, $P=0.005, R R=0.52(0.32-0.82))$ and the severe controls $\left(\chi_{1}^{2}=5.6, P=0.017\right)$. The DQw6 haplotype was significantly less frequent among the severe malarial anaemics compared with the mild controls $\left(\chi_{1}^{2}=4.9, P=0.026, R R=0.49(0.25-0.93)\right)$ but not compared with the severe controls $\left(\chi_{1}^{2}=0.1\right)$. All 96 Gambian haplotypes with DRB1*1302, analysed by PCR and oligonucleotide hybridization ${ }^{49}$ had the DRB3*0301 sequence ${ }^{50}$ (corresponding to the serological specificity DRw52c), as is true in other populations.

METHODS. The DRB1, DQA1 and DQB1 genes were analysed from an individual homozygous for the DRw13.02 RFLP pattern (Table 4) by PCR using locus-specific primers ${ }^{53}$ for exon 2, cloning by blunt-ended ligation into Smal-cut $\mathrm{m} 13 \mathrm{mp} 8$ vectors (Amersham) and dideoxy-sequencing. To type the DQB allele of all individuals with DRB1*1302, either a PCR or a Southern blot method was used. In the PCR method the second exon of the DQB genes was amplified using the primers 5'-CGCAGAGGATTTCGTGTACCAG- $3^{\prime}$ and 5'-GGGCGACGACGCTCACCTC-3' and hybridization was with oligonucleotides specific for DQB1*0501

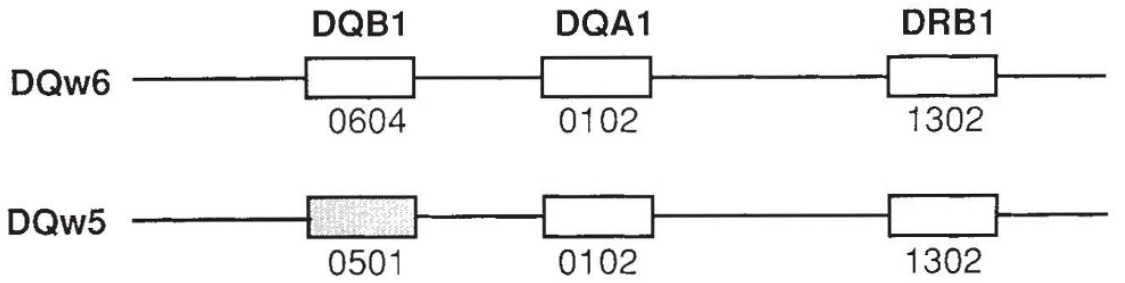

$\begin{array}{lcccccc}\text { Haplotype } & \begin{array}{c}\text { Severe malarial } \\ \text { anaemia } \\ n=436\end{array} & \begin{array}{c}\text { Cerebral } \\ \text { malaria } \\ n=826\end{array} & \begin{array}{c}\text { Mild } \\ \text { controls } \\ n=958\end{array} & \begin{array}{c}\text { Mild } \\ \text { malaria } \\ n=698\end{array} & \begin{array}{c}\text { Severe } \\ \text { controls } \\ n=660\end{array} & \begin{array}{c}\text { Healthy } \\ \text { adults } \\ n=408\end{array} \\ \begin{array}{l}\text { DRB1 }{ }^{\star} 1302- \\ \text { DQB1*0604 }\end{array} & 3.0 & 4.8 & 6.0 & 6.3 & 3.5 & 6.4 \\ \begin{array}{l}\text { DRB1*1302- } \\ \text { DRB }\end{array} & 5.7 & 8.7 & 10.5 & 7.6 & 10.0 & 10.3\end{array}$

(5'-CCGGGCAGTGACGC-3') or DQB1*0604 (5'-GAGGTGGGGTACCGC- $\left.3^{\prime}\right)$. The latter will also hybridize with the uncommon DQB1*0605 allele ${ }^{45}$ which, if present, will be included with ${ }^{*} 0604$ in this comparison. The Southern blot method employed the restriction enzyme BamHI and hybridization with a $\mathrm{DQB}$ probe which gave $7.5-\mathrm{kb}$ and $3.4-\mathrm{kb}$ bands with the DQw6 and DQw5 haplotypes, respectively ${ }^{48}$ 
immune response can be mounted may be small, a consideration of some importance for vaccine development.

Analysis of the mechanisms of the HLA associations described here may be helpful in identifying the antigens against which protective immune responses are directed. Although several $P$. falciparum antigens have been advocated or used as candidate vaccines, progress in vaccine development has been retarded by

TABLE 4 HLA class II haplotypes

\begin{tabular}{|c|c|c|c|c|c|c|c|c|c|}
\hline \multicolumn{2}{|c|}{ Serotype } & \multicolumn{2}{|c|}{$\begin{array}{l}\text { RFLP } \\
\text { pattern }\end{array}$} & \multirow{2}{*}{$\begin{array}{c}\text { Severe } \\
\text { malarial } \\
\text { anaernia }\end{array}$} & \multirow{2}{*}{$\begin{array}{c}\text { Cerebral } \\
\text { malaria }\end{array}$} & \multirow{2}{*}{$\begin{array}{c}\text { Mild } \\
\text { controls }\end{array}$} & \multirow{2}{*}{$\begin{array}{l}\text { Mild } \\
\text { malaria }\end{array}$} & \multirow{2}{*}{$\begin{array}{l}\text { Severe } \\
\text { controls }\end{array}$} & \multirow{2}{*}{$\begin{array}{c}\text { Healthy } \\
\text { adults }\end{array}$} \\
\hline DR & $\mathrm{DQ}$ & $\mathrm{DR}$ & $\mathrm{DQ}$ & & & & & & \\
\hline & & & & $n=436$ & $n=826$ & $n=958$ & $n=698$ & $n=660$ & $n=408$ \\
\hline 13.04 & 7 & $X X I$ & V & 24.8 & 26.4 & 25.3 & 30.4 & 27.1 & 23.8 \\
\hline 13.02 & 1 & $x$ & I & 8.7 & 13.5 & 16.4 & 13.9 & 13.5 & 16.7 \\
\hline 10 & 1 & III & 1 & 7.1 & 9.1 & 8.7 & 8.0 & 9.7 & 7.4 \\
\hline 9 & 2 & XVII & IX & 7.8 & 7.1 & 7.7 & 5.9 & 4.9 & 6.4 \\
\hline 4 & 8 & XII & IV & 5.7 & 4.4 & 5.2 & 4.6 & 4.4 & 4.7 \\
\hline 1 & 1 & 1 & 1 & 5.1 & 5.1 & 4.3 & 2.7 & 3.6 & 3.4 \\
\hline 11.01 & 7 & $x x$ & V & 2.8 & 2.5 & 3.4 & 3.9 & 3.5 & 3.2 \\
\hline 13.01 & 1 & IX & ॥ & 3.4 & 2.8 & 3.4 & 3.6 & 3.6 & 1.7 \\
\hline 8 & 7 & V & V & 3.2 & 2.7 & 4.0 & 3.4 & 3.0 & 3.0 \\
\hline 3.01 & 2 & VIII & III & 3.4 & 2.8 & 2.2 & 2.2 & 3.0 & 1.5 \\
\hline 7 & 2 & XIV & $\mathrm{VI}$ & 4.6 & 3.1 & 1.8 & 3.0 & 2.6 & 3.2 \\
\hline 13.03 & 7 & $X V$ & V & 2.5 & 2.4 & 2.0 & 2.0 & 1.8 & 2.9 \\
\hline 3.02 & 4 & $X V$ & IV & 1.6 & 2.2 & 1.0 & 2.4 & 2.9 & 2.2 \\
\hline
\end{tabular}

Percentage frequencies of the 13 common HLA class II haplotypes in the various groups. The total number of haplotypes typed in each group $(n)$ is shown (that is, twice the number of individuals). Haplotypes are identified by RFLP Jabels in Roman numerals ${ }^{46}$ and the corresponding serological types are shown with the subtype of the DRw3, DRw11 and DRw13 specificities appended after a decimal point. Analysis: The DRw 13.02-DQw1 haplotype was less frequent among cases of severe malarial anaemia than mild controls $\left(R R=0.49(0.33-0.72), x_{1}^{2}=14.0, P=0.00018, P_{\text {corrected }}=\right.$ 0.0023 ) but the reduction in frequency among the cerebral malaria group was not significant $\left(\chi_{1}^{2}=2.8\right)$. The DR7-DQw2 haplotype was more frequent among cases of severe malarial anaemia than among mild controls $\left(R R=2.66(1.32-5.38), \chi_{1}^{2}=8.1\right.$, $P=0.0044, P_{\text {corrected }}=0.057$ ). Phenotype frequencies were also analysed. For the DRw13.02 haplotype there were two homozygotes and 34 heterozygotes among the severe malarial anaemics, six homozygotes and 100 heterozygotes among the cerebral malarias, and 19 homozygotes and 120 heterozygotes among the mild controls. Thus, homozygotes for this haplotype had greater protection from severe malarial anaemia $(R R=0.22)$ than heterozygotes $\left(R R=0.55 ; \chi^{2}\right.$ for trend $=13.8,1$ d.f. $\left.P=0.0002\right)$ and homozygotes showed reduced risk of cerebral malaria $\left(R R=0.36(0.12-0.95), \chi_{1}^{2}=4.3\right.$, $P=0.04)$, but heterozygotes did not $(R R=0.95(0.69-1.31))$. Of the seven cases of severe malaria who were $\mathrm{HbS}$ carriers, two (one of three with cerebral malaria and one of four with severe malarial anaemia) were DRw13.02 heterozygotes. Logistic regression analysis by phenotype ${ }^{56}$, which allowed for possible confounding effects of differences in age, sex, ethnic group and area of residence between the groups of subjects, showed that the overall contribution of all 13 HLA class II haplotypes to differences between the severe malarial anaemic and mild control groups was statistically significant $\left(\chi^{2}=25.3,13\right.$ d.f., $P=0.02$ ). The adjusted relative risks for $D R w 13.02$ DQw1 and DRw7-DQw2 became, respectively, $0.45(0.27-0.72)\left(\chi_{1}^{2}=10.6, P=0.001\right)$ and $2.42(1.17-4.99)\left(\chi_{1}^{2}=5.7, P=0.02\right)$. The difference in HLA class I haplotype distribution between children with cerebral malaria and mild controls was close to significance $\left(\chi_{1}^{2}=22.5,14 \mathrm{df}, P=0.07\right)$ when the effect of homozygosity for DRw13.02DQw1 was included. The percentage frequencies of individuals homozygous for any TaqI DRB-DQB RFLP pattern in each group were: severe malarial anaemia, $6.9 \%$; cerebral malaria, $14.5 \%$; mild controls, $12.5 \%$; mild malaria, $13.5 \%$; severe controls, $17.0 \%$; healthy adults, $13.2 \%$. The homozygosity rate among the severe malarial anaemia cases was significantly lower than in the mild controls $\left(\chi_{1}^{2}=4.4, P=0.036\right)$ and severe controls $\left(\chi_{1}^{2}=11.0, P=0.001\right)$. RFLP analysis using Taql digestion and DRB- and $D Q B$-specific probes was used as the primary HLA class II typing method because, unlike serology, it requires only small amounts of blood and allows DRw13 subtypes to be distinguished and, unlike PCR-oligonucleotide typing methods, it should not miss new alleles in previously unstudied populations. Only the most frequent 13RFLP-defined haplotypes (all those $>2 \%$ frequency, accounting for about $85 \%$ of all haplotypes) were compared as the others were all too rare to allow a significant association to be detected unless the associated relative risk turned out to be improbably large $(R R>5)$ or small $(R R<0.2)$. The DRB and DQB band pattern labels in Roman numerals are as reported previously ${ }^{46,74,75}$. (XXI is identical to $V I$ except that the 4.1 -kilobase (kb) band is smaller, $4.0 \mathrm{~kb}$; XX is identical to XV except that the $11 \mathrm{~kb}$ band is $11.5 \mathrm{~kb}$; $X V I$ differs from XIV only in that the intense $4.0-\mathrm{kb}$ band is $4.1 \mathrm{~kb}$.) The usual complete associations of RFLP patterns with DR types and subtypes, defined by oligonucleotides $^{76}$, were confirmed for this population, except that the common XXI- $V$ haplotype was associated with both DRB1*1304 (78\% of alleles) and DRB1*1102 (manuscript submitted). The association of the X RFLP pattern with the DRB1*1302 allele was confirmed for all 48 haplotypes tested using allele-group specific amplification with the primers 5'-TCCTGGACAGATACTTCC- $3^{\prime}$ and $5^{\prime}$-GCACTGTGAAGCTCTCAC- $3^{\prime}$ followed by hybridization with the oligonucleotide probe $5^{\prime}$-ACATCCTGGAAGACGAGCG- $3^{\prime}$; these primers amplify DRB1*1302 but not DRB1*1301 and a positive signal on subsequent hybridization is specific for DRB1*1302. our ignorance of the nature of protective immunity in man. A search for an association between the ability to respond to a particular malaria antigen or epitope and the presence of protective HLA types may be useful in identifying one of these antigens as a target of natural protective immunity and thus a preferred antigen for vaccine trials.

\section{Evolutionary implications}

This study provides the clearest evidence yet obtained that a lethal infectious pathogen, one which has had enormous effects on human history ${ }^{17}$, is influencing the evolution of polymorphic $\mathrm{MHC}$ genes in man. Although a study of survivors of typhoid and yellow fever epidemics in Surinam ${ }^{58}$ suggested that HLA antigens may have influenced outcome in one or other of these diseases, only in Marek's disease of chickens ${ }^{59}$ had there been a convincing association with a fatal natural infection. These African data indicate that pathogen-driven selection is operating in present-day human populations and that it is unnecessary to propose $^{2}$ that this phenomenon has been episodic with long intervening periods of selective neutrality. Another implication is that HLA associations with many other potentially fatal infectious diseases may exist, but that very large sample sizes, with detailed molecular typing, may be necessary to demonstrate these convincingly.

The degree of protection afforded to children with the HLA types we have identified allows some estimates to be made of the time it would take for these alleles to reach their present-day frequencies under natural selection by malaria. Assuming simple directional selection this would take between 2,000 and 7,000 years for HLA-Bw53, depending on the initial allele frequency assumed $^{60}$ : estimates of the length of time $P$. falciparum malaria has been a major cause of mortality in Africa are similar ${ }^{17}$. However, we feel that selection pressures are unlikely to be this constant, and fluctuating selection pressures ${ }^{61-63}$ due to epidemics of infectious diseases might actually help to maintain MHC diversity. In this regard, two other models of how MHC diversity could be maintained by differential protection from infectious pathogens have been advocated: overdominant ${ }^{6,12}$ selection (heterozygote advantage) and frequency-dependent selection $^{64,65}$, in which individuals with rare alleles have the greatest resistance to pathogens. It is a prediction of the overdominance model that heterozygotes, who should be fitter than homozygotes, would be less common in the disease group than among controls. However, among cases of severe malarial anaemia, HLA class II haplotype heterozygotes were significantly more frequent than in the controls (Table 4), as were heterozygotes for HLA-B alleles in the severe malaria group compared with controls (Table 2). Furthermore, homozygotes for DRB1*1302 had lower relative risks than heterozygotes both for severe malarial anaemia and for cerebral malaria, which may also be more compatible with frequency-dependent than overdominant selection. Although the precision of this analysis is limited by the inability of the typing methods used to detect all true heterozygotes, and other infectious pathogens may show different HLA associations, these data do not support the recent tendency to assume that balancing selection for MHC genes must be overdominant ${ }^{11}$.

The importance of these HLA associations is emphasized by comparing the proportion of potential cases of severe malaria in the population which are prevented by the presence of HLABw53, DRB1*1302 or $\mathrm{HbS}$. Although $\mathrm{HbS}$ is clearly the most protective to an individual, and associated with a $92 \%$ reduction in the relative risk of severe malaria, only $13 \%$ of the Gambian population are carriers, so the proportion of potential cases prevented $^{66,67}$ is $12 \%$. HLA-Bw53 and the DRB1*1302 haplotype are less protective but they are much more prevalent, so that the proportion of potential cases prevented can be calculated to be $10.3 \%$ of severe malaria cases (HLA-Bw53) and $16 \%$ of cases of severe malarial anaemia (DRB1*1302): a total of about $15 \%$ of all potential severe malaria cases in The Gambia. 
Although mechanisms other than pathogen-driven selection might also contribute to the maintenance of MHC polymorphism, this study provides direct evidence that natural selection by infectious diseases exerts an important and continuing evolutionary pressure on HLA genes.

Received 31 May; accepted 16 July 1991

1. Dupont, B. (ed.) Immunobiology of HLA (Springer, New York, 1989).

2. Klein, J. Hum. Immunol. 19, 155-162 (1987).

3. Serjeantson S. W. Transplantation Proc 21, 598-601 (1989).

4. Lawlor, D. A., Ward. F. E., Ennis, P. D., Jackson, A. P. \& Parham, P. Nature 335, 268-271 (1988)

5. Figueroa, F., Gunther, E. \& Kiein, J. Nature 335, 265-267 (1988).

6. Takahata, N. \& Nei, M. Genetics 124, 967-978 (1990).

7. Hedrick, P. W. \& Thomson, G. Genetics 104, 449-456 (1983).

8. Klitz, W., Thomson, G. \& Baur, M. P. Am. J. hum. Genet. 39, 340-349 (1986).

9. Bjorkman, P. J. et al. Nature 329, 512-518 (1987).

10. Parham, P. et al. Proc. natn. Acad. Sci. U.S.A. 85, 4005-4009 (1988).

11. Hughes, A. L. \& Nei, M. Nature $\mathbf{3 3 5}, 167-170(1988)$.

12. Doherty, P. C. \& Zinkernagel, R. M. Lancet i, 1406-1409 (1975).

13. Tiwari, J. L. \& Terasaki, P. I. H.A and Disease Associations (Springer, New York, 1985).

14. Yamazaki, K. et al. J. exp. Med. 144, 1324-1335 (1976).

15. Clarke, B. \& Kirby, D. R. S. Nature 211, 999-1000 (1966)

16. Hedrick. P. W. \& Thomson, G. Genetics 119, 205-212 (1988).

17. Bruce-Chwatt, L. J. in Malaria Drinciples and Practice of Malariology 1-59 (eds Wernsdorfer, W. H. \& McGregor, I. A. Churchil1-Livingstone, Edinburgh, 1988)

18. Playfair. J. H. L. Br. med. Bull. 38, 153-159 (1982).

19. Schofield, L. et al. Nature 330, 664-666 (1987).

20. Weiss, W. R. et al. Proc. natn. Acad. Sci. U.S.A. 85, 573-576 (1988)

21. Hoffman, S. L. et al, Science 244, 1078-1081 (1989).

22. Romero. P. et al. Nature 341, 323-325 (1989).

23. Greenwood, B. M. Parasitol. Today 6, 188 (1990).

24. Greenwood. B. M. et al. Trans Roy. Soc. trop. Med. Hyg. 81, 478-486 (1987).

25. Allison, A. C. Cold Spring Harb. Symp. quant. Biol. 29, 137-149 (1964).

26. Sy, N. E., Long, G. W. Alcantara, A. K., Ranoa, C. P. \& Cross, J. H. Southeast Asian J. trop. Med. Public Health 15, 1-6 (1984).

27. Restrepo, M.. Rojas, W., Montoya, F., Montoya, A. E. \& Dawson, D. V. Rev. Inst. Med. trop. S. Paulo 30, 323-331 (1988).

28. Piazza, A. et al. Ann. hum. Genet. 49, 47-63 (1985)

29. Brown, P. J. Hum. Biol. 53, 367-382 (1981)

30. Brewster D Kwiatkowski, D \& White, N j Lancet 336, 313-319 (1990)

31. Warrell, D. A., Molyneux, M. E. \& Beales, P. F. Trans. Roy. Soc. trop. Med. Hyg. 84, suppl. 2, 1-65 (1990).

32. MacPherson, G. G. et al. Am. J. Path. 119, 385-401 (1988).

33. Bouharoun-Tayoun, H., Attanath, P., Sabchareon, A., Chongsuphajaisiddhi, T. \& Druilhe, P. J. exp. Med. 172, 1633-1641 (1990).

34. Allsopp. C. E. M. et al. Hum. Immun. 30, 105-109 (1991)

35. Hayashi, H. et al. Immunogenetics 32, 195-199 (1990)

36. Okoye R. C. et al. Tissue Antigens 25, 142-155 (1985)

37. Festenstein. H. et al, in Histocompatibility resting (eds Dausset, J. \& Colombani, J.) 175 178 (Munksgaard, Copenhagen, 1972).
38. Dutoit, E. D., Emmanuel, J. C., West, G., Taljaard, D. G. \& Oudshoorn, M. Tissue Antigens 36, 122-124 (1990)

39. du Toit, E. D., MacGregor, K. J.. Tal jaard, D. G. \& Oudshoorn, M. Tissue Antigens 31, 109-125(1987) 40. Aizawa, M. HLA in Asia-Oceania (Hokkaido University Press, Sapporo, 1986).

41. Baur, M. P. et al. in Histocompatibility Testing (eds Albert, E. D., Baur, M. P. \& Mayr, W. R.) 333-341 (Springer, Berlin, 1984)

42. Serjeantson, S. W. in The Colonization of the Pacific: a Genetic Trail (eds Hill, A. V. S. \& Serjeantson, S. W. 120-173 (Oxford University Press, Oxford 1989).

43. Cohen, S., McGregor, I. A. \& Carrington, S. Nature 192, 733-737 (1961).

44. Bodmer, J. et al. Tissue Antigens 37, 97-104 (1991)

45. Lee, K. W., Johnson. A. H. \& Hurley. C. K. J. Immun. 145, 3119-3125 (1990)

46. Carlsson, B., Wallin, J., Bohme, J. \& Motler, E. Hum. Immun 20, 95-113 (1987).

47. Bidwell, J. Immun, Today 9, 18-23 (1988).

48. Lee. K. W. Hurley, C. K. Hartzman, R. \& Johnson. A. H. Hum Immun 29, 202-219 (1990).

49. Fernandez-Vina, M., Shumway. W. \& Stastny. P. Hum. Immun. 28, 51-64 (1990)

50. Gorski, J. et al. J. exp. Med. 170, 1027-1032 (1989).

51. Schreuder, G. M. T. et al. in Immunobiology of HLA. (ed. Dupont, B.) 252-255 (Springer. New York, 1989).

52. Gyliensten, U. B., Lashikari, D. \& Ehrlich, H. A. Proc. natn. Acad. Sci. U.S. A. 87, 1835-1839(1990) 53. Scharf, S. J., Friedmann. A., Steinmann, L., Brautbar, C. \& Ehrlich, H. A. Proc. natn. Acad. Sci. U.S.A. 86, 6215-6219 (1989)

54. Ronningen, K. S. et al. Hum, immun, 29, 275-281 (1990)

55. Lee, T. D., Lee, G. \& Zhao, T M Tissue Antigens 35, 64-70 (1990)

56. Farewell, V. T. \& Dahlberg. S. Biometrics 40, 547-560 (1984)

57. Riley, E. M. Olerup, O. \& Troye-Blomberg, M. Parasitol. Today 7, 5-11 (1991)

58. de Vries, R. R.. Khan, P. M., Bernini, L. F., van Loghem. E. \& van Rood, J. J. J. Immunogenet. 6. 271-287 (1979)

59. Briles, W. E., Stone, H. A. \& Cole, R. K. Science 195, 193-195 (1977)

60. Hill, A. V. S. in Molecular Evolution of the Major Histocompatibility Complex (eds Klein, J. \& Klein, D.) (Springer, Berlin in the press).

61. Haldane, J. B. S. \& Jayakar, S. D. J. Genet. 58, 237-242 (1963).

62. Takahata, N. Genetics $\mathbf{9 8}, 427-440$ (1981).

63. Gillespie, J. H. Theor. Populat. Biol, 27, 222-237 (1985)

64. Snell, G. D. Folia biol, 14, 335-358 (1968).

65. Bodmer. W. F. Nature 237, 139-145 (1972)

66. Miettinen, O. S. Am. J. Epidem. 99, 325-332 (1974)

67. Bengtsson. B. O. \& Thomson, G. Tissue Antigens 18, 356-363 (1981)

68. Greenwood, B. M., Marsh. K. \& Snow, R. W. Parasitol. Today (in the press)

69. Molyneux, M. Taylor. T. E. Wirima, J. J. \& Borgstein, A. Q. J. Med. 72, 441-459 (1989)

70. Kwiatkowski, D. et at. Lancet 336, 1201-1204 (1990)

71. Kwiatkowski. D. \& Nowak, M. Proc. natn. Acad. Sci. U.S.A. 88, 5111-5113 (1991),

72. Colombo, B. \& Felicetti, L. J. med. Genet. 22, 291-292 (1985)

73. Hill, A. V. S. et at. Lancet 337, 640-642 (1991).

74. Martell, R. W., Oudshoorn. M., May, R. M. \& du Toit, E. D. Hum. Immun. 26, 237-244 (1989)

74. Martell, R. W., Oudshoorn, M., May, R. M. \& du Toit, E. D. Mum. Immun. 26, 237-244 (1989)

76. Wordsworth, B. P., Allsopp. C. E. M., Young, R. P. \& Bell, J. I. Immunogenetics 32, 413-418 (1980)

ACKNOWLEDGEMENTS. We thank the children and their parents and guardians who made this study possible; A. Hughes, V. Sarno, J. Wells, N. J. White, D. Walier, J. Crawley, S. Krishna, C. Craddock, U. Hughes, L. Bayo, L. Manneh, E. G. Sarr and T. Corrah for help with sample collection; M. Hassan-King, 1. Sambou, H. Sillah and F. Sisay for assistance in Fajara: C. Taylor and M. Bunce for assistance with serological typing: A. Ting and P. J. Morris for antisera and facilities; S. Abdalla, M. Shepherd and S. N. R. Yates for haemoglobin typing; O. Olerup and C. K. Hurley for discussions and communicating S. N. R. Yates for haemoglobin typing; O. Olerup and C. K. Hurley for discussions and communicating
their unpublished data; and J. I. Bell, T. E. A. Peto and D. J. Weatherall for encouragement and advice. A.V.S.H. is a Wellcome Trust Senior Clinical Fellow.

\section{LETTERS TO NATURE}

\section{An atomic switch realized with the scanning tunnelling microscope}

\section{M. Eigler, C. P. Lutz \& W. E. Rudge}

IBM Research Division, Almaden Research Center, 650 Harry Rd, San Jose, California 95120, USA

THE scanning tunnelling microscope ${ }^{1}$ (STM) has been employed in recent years in attempts to develop atomic-scale electronic devices, both by examining device-like characteristics in preexisting structures ${ }^{2,3}$ and by creating new structures by the precise manipulation of atoms and molecules with the STM tip ${ }^{4-6}$. Here we report the operation of a bistable switch that derives its function from the motion of a single atom. A xenon atom is moved reversibly between stable positions on each of two stationary conducting 'leads', corresponding to the STM tip and a nickel surface. The state of the switch is set (that is, the xenon atom is moved to the desired location) by the application of a voltage pulse of the appropriate sign across the leads. The state of the switch is identified by measuring the conductance across the leads. This switch is a prototype of a new class of potentially very small electronic devices which we will call atom switches.

The idea that an atom could be transferred from the tip of an STM to the surface by applying a sufficiently high voltage across the tunnel junction is due to Becker et al. ${ }^{7}$, who suggested that the atomic-scale perturbation left on a germanium surface was a germanium atom that had been transferred from the tip to the surface under high bias (tip negative) conditions. Subsequent work used static voltages or voltage pulses to make changes to the tip or to the surface being examined ${ }^{6,8-10}$. None of this work demonstrated that an atom can be transferred reversibly between a sample and tip, but Lyo and Avouris ${ }^{11}$ have recently been able to transfer silicon reversibly between a silicon surface and a tungsten STM tip.

As the conductance of a tunnel junction depends exponentially on the spacing between the electrodes (because the conduction-electron wavefunctions decay exponentially in the tunnel barrier), even slight rearrangements of the atoms in the currentcarrying region of the tunnel junction will lead to easily measured changes in the conductance. If controlled reversible motion of an atom between the electrodes of a tunnel junction could be achieved solely by the application of an electrical signal, an atomic-scale bistable electronic switch could be developed.

The atom switches that we will describe were constructed and operated using an STM contained in an ultra-high-vacuum system and cooled to $4 \mathrm{~K}$. The switches were constructed using the (110) surface of a single-crystal nickel sample and a tip made from polycrystalline tungsten wire. The sample was prepared by sputtering with argon ions and annealing to $820 \mathrm{~K}$. 